Sammlung Metzler

Band 246 


\title{
Einführung in die Literaturtheorie
}

4., erweiterte und aktualisierte Auflage

\author{
Aus dem Englischen \\ von
}

Elfi Bettinger und

Elke Hentschel

Verlag J.B. Metzler

Stuttgart - Weimar 
Die Originalausgabe erschien bei Basil Blackwell Publisher Limited, Oxford unter dem Titel "Literary Theory. An Introduction".

Second edition

(C) Terry Eagleton 1983, 1996

\author{
Für \\ Charles Swann \\ und \\ Raymond Williams
}

Die Deutsche Bibliothek - CIP-Einheitsaufnahme

Eagleton, Terry:

Einführung in die Literaturtheorie / Terry Eagleton. Aus dem Engl. von Elfi Bettinger und Elke Hentschel.

- 4., erw. und aktualisierte Aufl.

- Stuttgart ; Weimar : Metzler, 1997

(Sammlung Metzler ; Bd. 246)

Einheitssacht.: Literary theory $<\mathrm{dt}$. $>$

ISBN 978-3-476-14246-7

ISBN 978-3-476-14246-7

ISBN 978-3-476-04139-5 (eBook)

DOI 10.1007/978-3-476-04139-5

ISSN 0558-3667

SM 246

Dieses Werk einschließlich aller seiner Teile ist urheberrechtlich geschützt. Jede Verwertung außerhalb der engen Grenzen des

Urheberrechtsgesetzes ist ohne Zustimmung des Verlages unzulässig und strafbar. Das gilt insbesondere für

Vervielfältigungen, Übersetzungen, Mikroverfilmungen und die Einspeicherung und Verarbeitung in elektronischen Systemen.

(C) 1997 Springer-Verlag GmbH Deutschland

Ursprünglich erschienen bei J.B. Metzlersche Verlagsbuchhandlung und Carl Ernst Poeschel Verlag GmbH in Stuttgart 1997 


\section{Vorwort}

Wenn man den Ausgangspunkt der Veränderung, die die Literaturtheorie in diesem Jahrhundert erfahren hat, auf einen bestimmten Zeitpunkt festlegen wollte, so wäre das Jahr 1917, in dem der junge russische Formalist Viktor Šklovskij seinen bahnbrechenden Essay "Iskusstvo, kak priëm» (Die Kunst als Verfahren) veröffentlichte, keineswegs der schlechteste Vorschlag. Seitdem, und insbesondere in den beiden letzten Jahrzehnten, hat eine auffallende Bereicherung der Literaturtheorie stattgefunden: Die eigentliche Bedeutung von 'Literatur`, lesen` und iLiteraturkritik erfuhr einen tiefgreifenden Wandel. Aber bis auf einen kleinen Kreis von Spezialisten und Enthusiasten hat sich diese theoretische Revolution bisher noch nicht besonders weit verbreitet: ihre volle Auswirkung auf die Literaturstudenten/inn/en und die Leser/innen im allgemeinen steht noch aus.

Das vorliegende Buch soll eine einigermaßen verständliche Darstellung der modernen Literaturtheorie für diejenigen geben, die wenig oder gar keine Vorkenntnisse auf diesem Gebiet haben. Obgleich ein solches Vorhaben naturgemäß Auslassungen und unzulässige Simplifizierungen mit sich bringt, habe ich versucht, den Gegenstand eher 'populär und wissenschaftlich` allgemeinverständlich als im negativen Sinne populärwissenschaftlich darzustellen. Da es eine 'neutrale`, 'wertfreie‘ Darstellungsweise meiner Meinung nach nicht gibt, gebe ich meinen Standpunkt durchweg deutlich zu erkennen und hoffe, damit zusätzliches Interesse an dem Buch zu wecken.

Der Ökonom J. M. Keynes hat einmal bemerkt, daß diejenigen Wirtschaftswissenschaftler, die der Theorie ablehnend gegenüberstehen oder ohne sie besser zurechtzukommen behaupten, einfach noch einer älteren Theorie verhaftet sind. Das trifft auch für Literaturstudenten, -kritiker und -wissenschaftler zu. Manche beklagen sich, daß die Literaturtheorie unglaublich esoterisch sei - vermuten darin eine geheimbündlerische, elitäre Enklave, die der Nuklearphysik ziemlich ähnlich ist. Zwar ermutigt literarische Bildung nicht gerade zum analytischen Denken; aber Literaturtheorie ist in Wirklichkeit nicht schwieriger als viele andere theoretische Untersuchungen, und sogar um einiges einfacher als manche von ihnen. Ich hoffe, daß das vorliegende Buch einen Beitrag dazu leistet, denen die Angst zu nehmen, die befürchten, daß der Gegenstand ihre Verständnisfähigkeit überschreitet. Einige Student/inn/en und Kritiker/inn/en verwahren sich auch dagegen, daß die Literaturtheorie zwischen den Leser und 
das Werk strete. Die einfache Antwort darauf ist, daß wir ohne irgendeine Art von Theorie, wie unreflektiert und unbewußt sie auch immer sein mag, gar nicht erst wüßten, was überhaupt ein literarisches Werk ist oder wie wir es lesen sollen. Eine feindselige Einstellung der Theorie gegenüber bedeutet normalerweise eine Ablehnung der Theorien anderer und ein Übersehen der eigenen. Ein Anliegen dieses Buches besteht darin, diese Verdrängung aufzuheben und uns ein Wiedererkennen zu ermöglichen.

T.E.

Im Einverständnis mit dem Autor und dem englischen Verlag ist die vorliegende deutsche Ausgabe um das Kapitel "The Rise of English" gekürzt, das den Aufstieg und die Entwicklung der Literaturwissenschaft in Großbritannien und in den USA behandelt. Die dort enthaltene grundsätzliche Auseinandersetzung mit dem New Criticism wurde jedoch beibehalten und an den Beginn des Kapitels über "Strukturalismus und Semiotik" gestellt. Die Anmerkungen der englischen Ausgabe wurden prinzipiell in den Text eingearbeitet. Das Literaturverzeichnis enthält alle vom Autor benutzten Titel (mit den entsprechenden deutschen Ausgaben) sowie weiterführende Literatur für die deutschen Leser und Leserinnen.

\section{Vorwort zur zweiten Auflage}

Dieses Buch ist ein Versuch, die moderne Literaturtheorie einer möglichst großen Leserschaft verständlich und attraktiv zu machen. Mit Befriedigung kann ich berichten, daß es seit seiner ersten Veröffentlichung 1983 von JuristInnen wie LiteraturwissenschaftlerInnen, von AnthropologInnen ebenso wie von KulturtheoretikerInnen studiert worden ist. In einer Hinsicht ist das vielleicht gar nicht so überraschend: Wie das Buch selbst zu zeigen versucht, gibt es in der Tat gar keine 'Literaturtheories im Sinne eines Korpus von Theorien, das nur der Literatur entspringt oder das allein auf Literatur anzuwenden wäre. Keiner der hier in Umrissen vorgestellten Ansätze, von der Phänomenologie und der Semiotik bis hin zum Strukturalismus und der Psychoanalyse, ist nur mit literarischen Texten befaßt. Ganz im Gegenteil sind alle aus anderen geisteswissenschaftlichen Gebieten hervorgegangen und ihre Implikationen weisen über die Literatur hinaus. Ich denke, daß dies einer der Gründe für die Popularität des Buchs ist, und auch ein Grund, warum sich eine 
Neuauflage lohnt. Aber ich bin auch von der Anzahl nicht-akademischer Leser beeindruckt, die es angesprochen hat. Anders als den meisten solcher Bücher ist es ihm gelungen, eine Leserschaft jenseits der akademischen Institutionen zu erreichen, was besonders angesichts des sogenannten elitären Anspruchs von Literaturtheorie interessant ist. Wenn es eine schwierige, ja sogar esoterische Sprache ist, dann scheint es eine zu sein, die auch Menschen interessiert, die nie eine Universität von innen gesehen haben; und wenn das so ist, dann sollte das diejenigen, die sie innerhalb der Universität als esoterisch abtun, nochmals zum Nachdenken bewegen. Es ermutigt jedenfalls, daß es in einem postmodernen Zeitalter, in dem von Bedeutung wie von allem anderen sofortige Konsumierbarkeit erwartet wird, Menschen gibt, die es für lohnend halten, sich eine neue Art, Möglichkeit, über Literatur zu sprechen, zu erarbeiten.

Manche Arten von Literaturtheorie sind tatsächlich in übertriebener Weise obskurantistisch und nur Eingeweihten zugänglich, und dieses Buch versucht, den angerichteten Schaden zu beheben und einen breiten Zugang zu ermöglichen. Aber auch noch in einem ganz anderen Sinne ist Literaturtheorie das genaue Gegenteil von elitär. Das am Literaturstudium wirklich Elitäre liegt in der Vorstellung, daß nur Menschen mit einer ganz bestimmten kulturellen Bildung literarische Werke schätzen könnten. Es gibt solche, die ,literarische Werte im Blut haben, und solche, die draußen in der Dunkelheit schmachten. Ein wichtiger Grund für die Entwicklung der Literaturtheorie seit den 60er Jahren war das allmähliche Zusammenbrechen solcher Auffassungen unter dem Einfluß einer neuen Studentenschaft aus angeblich unkultivierten' Gesellschaftskreisen, die in den Bereich der höheren Bildung eindrangen. Theorie war ein Weg, literarische Werke aus dem Würgegriff einer literarischen Sensibilität zu befreien und sie für eine Analyseart zu öffnen, an der sich zumindest prinzipiell jeder beteiligen konnte. Diejenigen, die sich über die schwierige Theorie beklagen, würden ironischerweise wohl kaum erwarten, ein Lehrbuch für Biologic oder für chemische Verfahrenstechnik sofort zu verstehen. Weshalb sollte dann das Literaturstudium anders sein? Vielleicht, weil wir erwarten, daß Literatur selbst eine einfache Art von Sprache ist, jedem sofort zugänglich; aber das stellt an sich bereits eine ganz bestimmte 'Theorier der Literatur dar. Richtig verstanden ist $\mathrm{Li}$ teraturtheorie eher von demokratischen als von elitären Anstößen geprägt worden; und in dem Grad, in dem sie wirklich in schwülstige Unlesbarkeit verfällt, wird sie ihren eigenen Wurzeln untreu.

T.E. 


\section{Inhalt}

Vorwort $\ldots \ldots \ldots \ldots \ldots \ldots \ldots \ldots \ldots \ldots, \mathrm{V}$

Vorwort zur zweiten Auflage .............. VII

1. Einleitung: Was ist Literatur? . . . . . . . . . 1

2. Phänomenologie, Hermeneutik, Rezeptionstheorie ..................... 19

3. New Criticism, Strukturalismus und Semiotik........................ 59

4. Der Poststrukturalismus .............. 110

5. Die Psychoanalyse $\ldots \ldots \ldots \ldots \ldots \ldots \ldots .138$

Schluß: Politische Kritik . . . . . . . . . . . 187

Nachwort...................... 214

Bibliographie ..................... 239

Register ....................... 254 